\title{
Dynamic self-organization in holonic multi-agent manufacturing systems: The ADACOR evolution
}

\author{
José Barbosa ${ }^{\mathrm{a}, \mathrm{c}, \mathrm{d}, *}$, Paulo Leitão ${ }^{\mathrm{a}, \mathrm{b}}$, Emmanuel Adam ${ }^{\mathrm{c}, e}$, Damien Trentesaux ${ }^{\mathrm{c}, \mathrm{d}}$ \\ a Polytechnic Institute of Bragança, Campus Sta Apolónia, Apartado 1134, 5301-857 Bragança, Portugal \\ b LIACC - Artificial Intelligence and Computer Science Laboratory, R. Campo Alegre 102, 4169-007 Porto, Portugal \\ ${ }^{\mathrm{C}}$ Univ. Lille Nord de France, F-59000 Lille, France \\ ${ }^{\mathrm{d}}$ UVHC, TEMPO Research Center, F-59313 Valenciennes, France \\ e UVHC, LAMIH, F-59313 Valenciennes, France
}

\section{A R T I C L E I N F O}

\section{Article history:}

Received 12 October 2013

Received in revised form 29 October 2014

Accepted 29 October 2014

Available online 20 November 2014

\section{Keywords:}

Holonic manufacturing systems

Multi-agent systems

Evolutionary systems

Self-organization

\begin{abstract}
A B S T R A C T
Nowadays, systems are becoming increasingly complex, mainly due to an exponential increase in the number of entities and their interconnections. Examples of these complex systems can be found in manufacturing, smart-grids, traffic control, logistics, economics and biology, among others. Due to this complexity, particularly in manufacturing, a lack of responsiveness in coping with demand for higher quality products, the drastic reduction in product lifecycles and the increasing need for product customization are being observed. Traditional solutions, based on central monolithic control structures, are becoming obsolete as they are not suitable for reacting and adapting to these perturbations. The decentralization of the complexity problem through simple, intelligent and autonomous entities, such as those found in multi-agent systems, is seen as a suitable methodology for tackling this challenge in industrial scenarios. Additionally, the use of biologically inspired self-organization concepts has proved to be suitable for being embedded in these approaches enabling better performances to be achieved. According to these principals, several approaches have been proposed but none can be truly embedded and extract all the potential of self-organization mechanisms. This paper proposes an evolution to the ADACOR holonic control architecture inspired by biological and evolutionary theories. In particular, a two-dimensional self-organization mechanism was designed taking the behavioural and structural vectors into consideration, thus allowing truly evolutionary and reconfigurable systems to be achieved that can cope with emergent requirements. The approach proposed is validated with two simulation use cases.
\end{abstract}

(C) 2014 Elsevier B.V. All rights reserved.

\section{Introduction}

Nowadays, manufacturing systems face unprecedented challenges imposed by highly demanding constraints ranging from high product customization to the demand for lower prices as well as increasing product quality to significant fluctuations in market demands [1]. Current manufacturing systems, based on traditional hierarchical and rigid control structures, can no longer cope with these demanding constraints [2,3], and require the use of new manufacturing paradigms that meet these constraints better. In

\footnotetext{
* Corresponding author at: Polytechnic Institute of Bragança, Campus Sta Apolónia, Apartado 1134, 5301-857 Bragança, Portugal. Tel.: +351 918985512.

E-mail addresses: jbarbosa@ipb.pt (J. Barbosa), pleitao@ipb.pt (P. Leitão), emmanuel.adam@univ-valenciennes.fr (E. Adam),

damien.trentesaux@univ-valenciennes.fr (D. Trentesaux).
}

this sense, to respond more quickly and efficiently, manufacturing systems are shifting to novel paradigms composed of features such as modularity, scalability, reconfigurability, robustness and flexibility, to name a few. Despite the benefits that these features bring, new control structures also need to be developed in parallel and all the potential extracted from the manufacturing paradigms.

Traditionally, manufacturing control systems use hierarchical control structures which concentrate the processing power of a shop-floor control under one central node. They improve performance and optimization, but respond inadequately to changes in conditions, scalability and unpredictability. These monolithic, rigid control structures are insufficient to meet the current requirements imposed by manufacturing environments which demand flexibility, robustness, reconfigurability and responsiveness. New manufacturing paradigms have thus emerged of which the common denominator is the decentralization and distribution of processing power over several entities, but with a decrease in 
system performance regarding process optimization. Examples of such paradigms are Reconfigurable Manufacturing Systems (RMS) [4], Multi-agent Systems (MAS) [5], Bionic Manufacturing Systems (BMS), Holonic Manufacturing Systems (HMS) [6], and more recently, Evolvable Production Systems (EPS) [7].

RMS is a concept that suggests the rapid change in the factory's structure using changes in hardware and/or software to adjust the production capacity and functionality quickly [8]. A RMS system should exhibit the following characteristics [4]: modularity, integrability, customization, convertibility and diagnosability. A MAS [5,9] is both a paradigm and technology that advocates the design of systems based on societies of decentralized, distributed, autonomous and intelligent entities, called agents. In such systems, each agent has a partial view of the surrounding world and must therefore cooperate with others in order to achieve the global objectives; the behaviour of the global system emerges from the cooperation between individual agents. An HMS [6] is a paradigm that translates the concepts of living organisms and social organizations developed by Koestler [10] to the manufacturing world. A holon, as Koestler coined the term, is an identifiable part of a system that has a unique identity, yet is made up of subordinate parts and is in turn part of a larger whole. Koestler also defines the term holarchy as a hierarchically organized system populated with self-regulating holons, and the system goals are achieved by the cooperation between holons. An HMS is the encapsulation of the entire manufacturing system in a holarchy. The holons can represent physical resources and logic entities.

MAS technology and/or HMS concepts have been successfully developed and applied to different domains (see for example the reviews (Leitao et al., 2013; [11])). MetaMorph [12] and its successor MetaMorph II [13] were projects that firstly aimed to provide an agent-based approach for the creation and management of agent communities in distributed manufacturing environments, and secondly to integrate cross-enterprise activities such as design, planning and scheduling. AARIA (Autonomous Agents at Rock Island Arsenal) was developed in the early years of agentbased architectures for military production, with the particularity of using internet as a means of communication between agents [14]. One of the best-known practical implementations using multi-agent systems is probably on one of the Daimler Chrysler production lines ([15]; [16]). This architecture aimed at using agent technology for both dynamic and flexible transportation systems and control systems.

One of the most remarkable HMS architectures is the PROSA (Product-Resource-Order-Staff Architecture) reference architecture that defines the main guidelines for developing a generic manufacturing control layer [17]. A real application of PROSA was conducted at Cambridge University using a packaging cell [18], where a collaboration was formed between order and resource holons to accommodate clients' demands. Order holons use negotiation techniques to ensure fast and reliable production and are also responsible for tracking production progress. On the other hand, the main aim of resource holons is to maximize the return on the execution of their services, and finally, product holon deal with the buying and selling of goods.

The ADACOR (ADAptive holonic COntrol aRchitecture for distributed manufacturing systems) [19] is a holonic architecture that proposes an adaptive production control approach balances from a stationary state to a transient state, in normal and unexpected conditions, respectively, combining the benefits of hierarchical and heterarchical control structures using an adaptive mechanism. Complementarily, a deeper and up-to-date description of the current holonic and multi-agent systems solutions can be found in the surveys of [20-22,23], where a more detailed description of the aforementioned and other not mentioned solutions is made.
To sum up, the existence of one central node controlling the low-level entities in the hierarchical approaches constitutes a drawback in the sense that if it fails the whole system may fail. On the other hand, decentralized systems, such as those elaborated using MAS and HMS concepts, respond better to perturbations where the failure of an isolated entity only affects part of the system, and the other parts can continue operating with no major impact. Despite the benefits shown, decentralized systems do not attain optimization levels as high as those depicted by hierarchical solutions. As illustrated in Fig. 1, under normal conditions, system performance of hierarchical architectures is better than heterarchical architectures. However, in unexpected situations, e.g. due to a resource malfunction or a rush order, the heterarchical architectures behave better as they are able to respond promptly to perturbations.

Essentially, the challenge is to combine the best of both worlds, where a system displays the optimization levels of hierarchical systems under normal conditions and behaves like heterarchical approaches in unexpected situations. An approach like this brings hierarchical features to distributed entities whilst retaining their autonomy. For this purpose, some hybrid solutions have been developed exhibiting $h a(t)$ behaviour illustrated in Fig. 1. ADACOR [19] is a well-known example of such an approach as it considers an adaptive production control mechanism that balances between two states: a hierarchical stationary state and a heterarchical transient state. In spite of the important progress made in this field, further developments must be made to achieve a truly dynamic and evolvable system that is able to cope with system constraints, without significantly affecting its functioning.

Biology and nature, as well as chaos and evolutionary theories are suitable sources of inspiration to design and develop solutions for solving complex, large-scale problems aimed at increasing their potential by embedding emergent concepts such as self-organization [24]. One example is the use of self-organization principles, which can be described as the ability of a system to arrange itself autonomously and spontaneously, mainly due to internal interactions, and without the need to use a central authority [25]. Among the most known biological sources of inspiration, one can find food foraging of ants [26] or food foraging of bees [27] as well as fish schooling or birds' flying pattern [28].

Some approaches have already tried to use self-organization concepts as a way to cope with the complexity and unpredictability associated with disturbances that may appear in the system. Some examples: the PROSA architecture was extended using food foraging behaviour of ants as a forecasting methodology [29]; the $\mathrm{P} 2000+[15]$ used a virtual buffer mechanism in machines that act as the self-organization regulator; ADACOR where the perturbation propagation uses a pheromone spreading technique as a warning signal among entities, which can assess the impact of the perturbation on themselves [19].

However, these biologically inspired mechanisms are only considered very lightly, lacking truly evolutionary concepts as a way to handle complex systems properly, e.g. minimizing the impact of disturbances. In this way, this paper proposes an

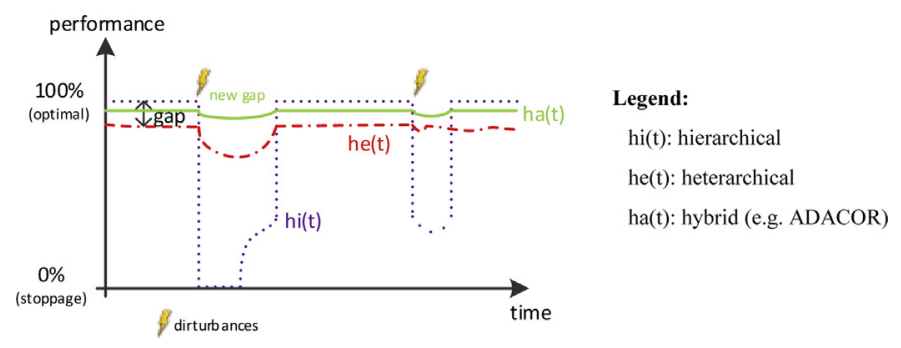

Fig. 1. Performance behaviour of different classical control structures. 
evolution to the ADACOR holonic manufacturing control architecture, by taking knowledge of biology, the chaos theory and the evolutionary theory into consideration to unleash the two predefined working states of its previous version by allowing the system to dynamically evolve using self-organization principles. For this purpose, ADACOR $^{2}$ reuses the ADACOR principles, mainly the holonic concepts and adaptive production control, enhanced with a two-dimensional self-organization model that allows intelligent complexes to smoothly or dramatically respond to new system constraints in such a way that the overall system performance is not degraded.

The paper is organized as follows: Section 2 overviews the main concepts of the ADACOR holonic manufacturing control architecture, and Section 3 presents the inspiration and core foundations of the $\mathrm{ADACOR}^{2}$ holonic control architecture, focusing mainly on the two-dimensional self-organization model. Sections 3.1 and 3.2 detail the two self-organization components, first at micro-level, named behavioural self-organization, and then at macro-level, called structural self-organization. Section 4 presents the details of self-organization model. Section 5 presents and discusses the experimental results from the application of the proposed selforganized holonic approach to real experimental use cases, and finally Section 6 rounds-up the paper with conclusions and future work.

\section{Basics of the ADACOR holonic manufacturing control architecture}

The ADACOR adaptive holonic architecture intends to combine the best practices of hierarchical and heterarchical control approaches, being as decentralized as possible and as centralized as necessary, i.e. using a centralized approach when the objective is optimization, and a more heterarchical approach in the presence of unexpected events and modifications [19]. In these circumstances, ADACOR proposes the decomposition of manufacturing control functions into a community of autonomous and cooperative holons, taking advantage of modularity, decentralization, agility, flexibility, robustness and scalability. To achieve this, ADACOR proposes four types of holons:

- Product holon $(\mathrm{PH})$, representing the products available in the factory plant catalogue and the knowledge to produce them.

- Task holon $(T H)$, responsible for managing the real-time execution of production orders on the shop floor.

- Operational holon $(\mathrm{OH})$, representing the system resources, e.g. robots and operators, responsible for governing its own agenda as well as managing the physical connection with the real resource.

- Supervisor holon $(\mathrm{SH})$, responsible for introducing optimization into the system.

ADACOR clearly defines the behaviour of individual holons using the Petri nets [30] formalism and also the interaction patterns between them using Agent UML [31] sequence diagrams. This is of great importance in the way that ADACOR proposes a binary-state balance for the adaptive production control depending on the system perturbation level, combining the benefits of hierarchical and heterarchical control structures using an adaptive mechanism.

As illustrated in Fig. 2, in stationary state, the holons are organized in a hierarchical structure, with supervisor holons playing the role of coordination and optimizing the schedules of their subordinates organized in clusters. This system runs in this configuration until a perturbation is detected. The operational holon that detects the disturbance (in this case $\mathrm{OH}_{1}$ ) then tries to recover locally by carrying out a self-diagnosis. If recovery from the failure is unsuccessful, its autonomy factor is increased and propagates the need for re-organization to the other holons in the system. The propagation mechanism involves depositing a pheromone on the neighbouring supervisor holon and its subsequent spread to other SHs.

The other holons that sense the pheromone from supervisor holons, increase their autonomy factors according to the pheromone's intensity and their local knowledge, and propagate the
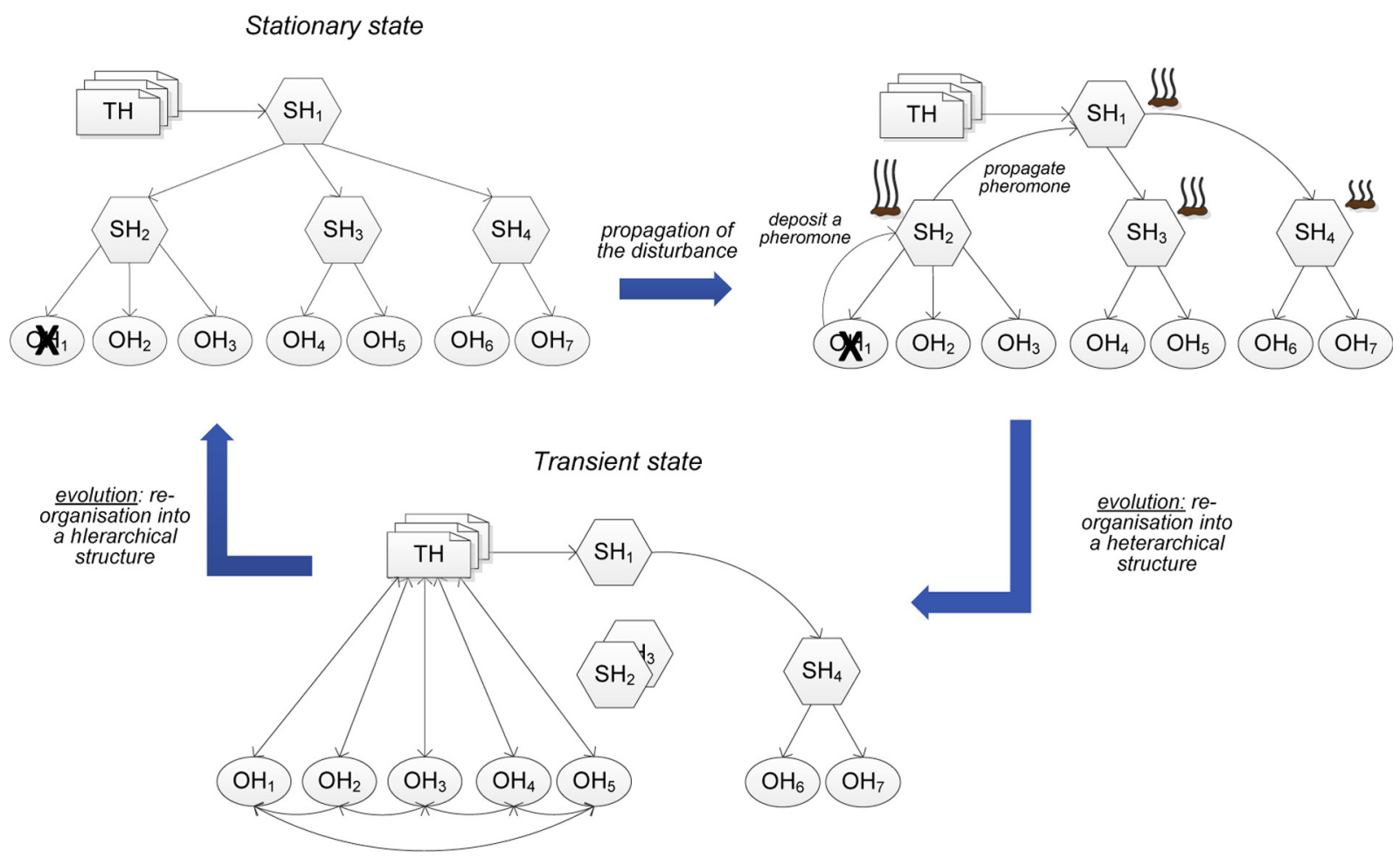

evolution: re-

organisation int

a heterarchical structure

Fig. 2. Adaptation mechanism in ADACOR supporting a hybrid control architecture. 
emergent re-organization to the neighbouring supervisor holons. The intensity of the odour associated with the pheromone decreases as the levels of supervisor holons increases (it is similar to the distance in the original pheromone techniques), according to a defined flow field gradient. Each individual holon, taking its autonomy factor, learning capabilities and pheromone intensity into consideration, decides if it should re-organize or not. In Fig. 2, the holons $\mathrm{OH}_{1}$ to $\mathrm{OH}_{5}$ choose to re-organize, while holons $\mathrm{OH}_{6}$ and $\mathrm{OH}_{7}$ do not re-organize as the pheromone's intensity is not high enough, i.e. the epicentre of the disturbance is far away and the impact is too low or zero.

In this transitory state, the task holons interact directly with the operational holons to achieve an alternative schedule in a short time, gaining in responsiveness. During this state, the supervisor holons continue elaborating optimized schedules, but now only the holons with low autonomy factors will accept the proposals. The holons remain in transient state during the reestablishment time, $\tau$, which is typically a short period of time. Once this time has elapsed, they verify if the pheromone odour has dissipated or is still active. If the pheromone is still active, the holons stay in transient state for an additional reestablishment time, until the pheromone has dissipated.

Once the pheromone has dissipated, each individual holon reduces its autonomy factor again and returns to a hierarchical control structure, going back to a stationary state, since they accept the schedules once more from their $\mathrm{SH}$. At this moment, the supervisor holons collect the updated individual schedules, achieved during the transient state, and proceed with the synchronization and posterior optimization of the existing schedule. The re-schedule is sent to the operational holons, which accept the advised schedule since they have a low autonomy factor again.

This powerful mechanism allows the system to respond quickly to perturbations and balance back to a stationary state after its dissipation, fulfilling the challenge of developing a hybrid control system, i.e. the $h a(t)$ curve in Fig. 1. Despite the potential and innovation introduced by embedding this self-organization mechanism, the control system only balances between two predefined states and is not able to evolve to other pre-defined or new control structures, supporting a truly evolvable and reconfigurable system. This challenge is in line with the current emergent, strong requirements, demanding an additional step taking the knowledge of biology, the chaos theory and the evolutionary theory into consideration to achieve the dynamic evolution and adaptation of manufacturing systems.

\section{Self-organization perspective in $\operatorname{ADACOR}^{2}$}

The $\mathrm{ADACOR}^{2}$ holonic architecture aims to enhance the existing ADACOR holonic architecture through self-organization and chaos principles to achieve a truly evolvable and reconfigurable system.

The proposed architecture allows the system to find a multitude of dynamic configurations, instead of the two fixed ones defined in ADACOR, based on the inspiration of two distinct evolutionary theories. Probably the most renowned evolutionary theory was developed in the observations and studies by Charles Darwin which were published in the book entitled "The Origin of Species" [32]. In this book, Darwin states that overtime species tend to make small internal changes in order to adapt to their environment (Fig. 3b). On the contrary, the punctuated equilibrium theory states that species tend to be in a stable state for long periods of time and suddenly make a drastic change [33], illustrated in Fig. 3, curve a. Similar concepts can be found in the manufacturing world where the kaizen philosophy states that the system can improve through small continuous changes while the kaikaku philosophy considers that drastic changes improve system performance [34].

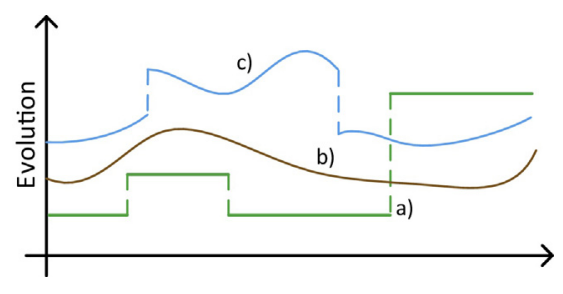

Fig. 3. Darwin and punctuated evolution theories.

These two dramatically different approaches, found either in evolutionary theories or manufacturing strategies, offer insights to respond agilely to unexpected events. With this in mind, this work proposes a two-dimensional self-organization model taking mechanisms at micro and macro level into consideration, as illustrated in Fig. 4, allowing the combination of smooth and drastic system evolution (illustrated in Fig. 3, curve c).

Behavioural self-organization is observed at micro level, where each individual holon can change its behaviour according to the external conditions resulting in a smooth evolution. The second component, named structural self-organization, is observed at a higher level and makes the system evolve drastically by changing the relationships between the holons (and even the cluster formation). In the next section, each of these components, as well the inter-dependencies between the two levels will be detailed further.

\subsection{Acting at micro level: behavioural self-organization}

Behavioural self-organization contributes at micro level to the global self-organization found in the $\mathrm{ADACOR}^{2}$ architecture. This self-organization component, seen as a way of helping the smooth evolution of the system to face unexpected changes in conditions, is achieved by providing the holons with a catalogue of behaviours and a behaviour selection engine.

For this purpose, each holon continuously monitors its state and its environment, seeking an opportunity to evolve, as well as being aware of any external evolution trigger. Depending on the trigger type, the holon self-organizes accordingly by selecting the right behaviour from the set of known behaviours. This selection is accomplished when the adequate behaviour is used at the exact moment with the lowest impact on the overall system. This type of self-organization will push the system to a new operating point, i.e. a new configuration, produced by small internal changes to cope with new constraints.

In this sense, the behavioural self-organization of $\mathrm{ADACOR}^{2}$ is defined as "the change in the internal state of the holon using a set of internal rules and mechanisms, triggered in response to a plan deviation or a new evolution opportunity, with the aim of reestablishing normal functioning or improving performance."

From a behavioural self-organization perspective, a plan deviation is detected or evolution opportunity discovered by

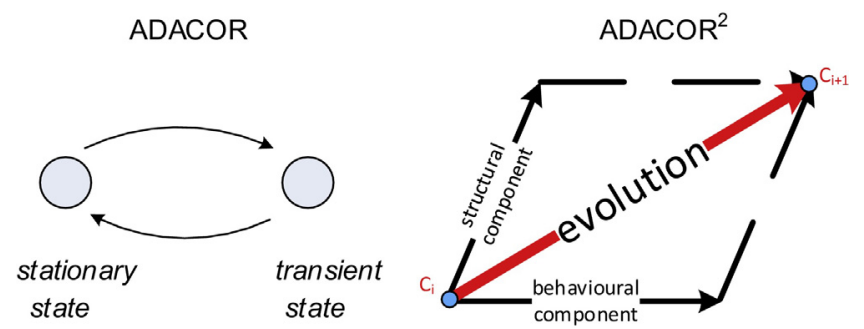

Fig. 4. $\mathrm{ADACOR}^{2}$ evolutionary perspective based on the two-dimensional selforganization model. 
matching a set of rules, present in a decision rule engine, with the information available.

The decision making process is accomplished using global data from $\mathrm{ADACOR}^{2}$ high level holons (if available and/or needed), namely the $\mathrm{SH}$, and local data from holons' local knowledge. In this way, the detection of previous situations, using the monitoring module, is sustained by means of access to global and local information. Access to the global information, related to the most optimized functioning, guarantees that behavioural decisions take into account broader, long-term solutions, thus decreasing the need for a new behavioural adaptation in the short term. On the other hand, local information supports the system reactivity by allowing the entity to access local data that at the moment of decision is more accurate, i.e. more up-to-date. Despite the shorter refresh rate of this data, it suffers from myopia in the sense that it represents a local, partial view for a smaller area of action [35], and so global data is used whenever possible.

The delicate part of finding the right balance between global and local data concerns the share given to each part, taking data reliability into consideration. Concerning this, at the start, when no reliable data information is available, the holons decide on equal shares, which will be refined (e.g. using reinforcement mechanisms) during its lifecycle.

A practical example of behavioural self-organization in action is depicted in Fig. 5. During the normal operational process, a scheduled order cannot be processed by the assigned resource. In this case, the $\mathrm{TH}$ responsible for managing the order will, using its set of known behaviours, adapt by changing its behaviour, rescheduling the order (which possibly affects the rest of the schedule).

In this example, the hierarchy formed within $\mathrm{SH \# 2}$ was dissolved and the $\mathrm{TH}$ reassigned the upcoming work orders to the available $\mathrm{OHs}$, some being part of the work orders assigned to the SH\#1 hierarchy.

\subsection{Acting at macro level: structural self-organization}

Structural self-organization presents a means of drastic evolution, re-arranging the relationships between the holons and/or their organization. Formally, in this work, structural selforganization can be defined as "the change in the relationships between holons, and consequently the change in the holarchy structure, which is triggered in response to a deep impact plan deviation, thus promoting a drastic response that aims to re-establish normal system functioning or improve its performance". This property can be divided into different levels, also linked to behavioural self-organization. On this subject, a three-level classification has been developed as follows:

- Level 0 (emergence): relations between holons are changed as a consequence of behavioural self-organization. This happens as a change in holon behaviour can imply a change in its relations, such as a change in the resource that will process the next operation. This level of structural change is classified as weak since the structural change is not directly driven by the need.

- Level 1 (logical structural self-organization): each holon is constantly trying to optimize its place in the holarchy structure. This constant optimization may drive the holon to change holarchy, belong to several holarchies at the same time or act as a freelancer to work completely autonomously.

- Level 2 (physical structural re-organization): similar to level 1 with the addition that holons, e.g. OHs, can physically change their place, changing not only their relations and positions in the holarchy but also their physical position.

Particularly, the last two levels, level 1 and 2, are related to the concepts envisioned by the RMS paradigm of rapid change at shopfloor level. In fact, in level 1 , one can assist to the logical reorganization on the control layer, which is mapped into the software change of the RMS paradigm and, in level 2, a hardware change is achieved by the physical re-arrangement of the resources present at the shop-floor.

The stimulus that can trigger a structural self-organization depends on the impact on the system and can be any event that disrupts or deviates the predicted execution of the system, such as a rush order, an order cancellation, a production quality issue, a supply shortage, or a resource malfunction. In order to illustrate this, three examples are provided in which a structural selforganization could be initiated. The first is the introduction of a big batch order; the second is the constant production equilibrium between two products, each one having particular requirements, and a third with resource sharing due to a malfunction on a similar resource. Assuming that the system is functioning in a stable state in a given configuration, and that a very large order arrives in the

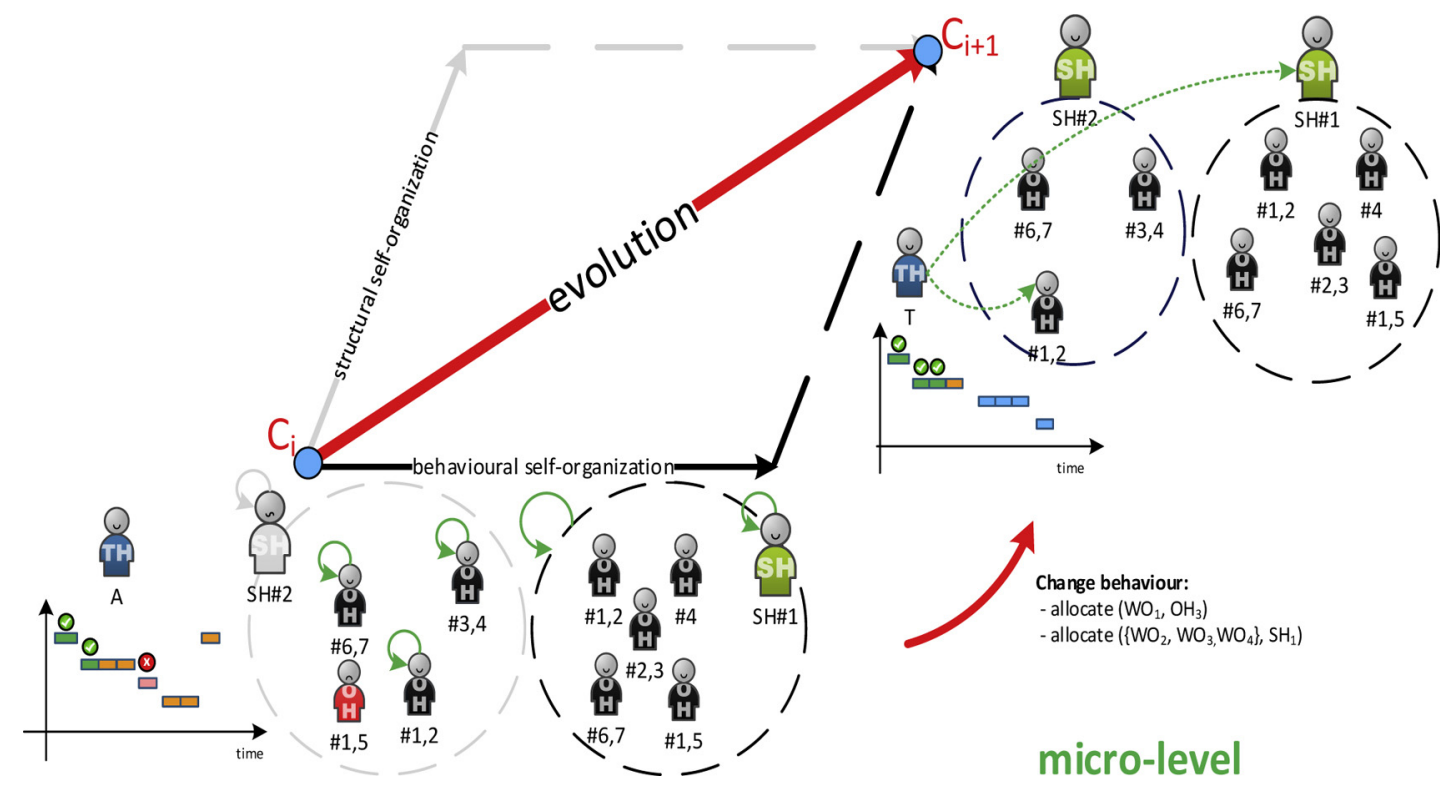

Fig. 5. Evolution by means of the self-organization mechanism. 


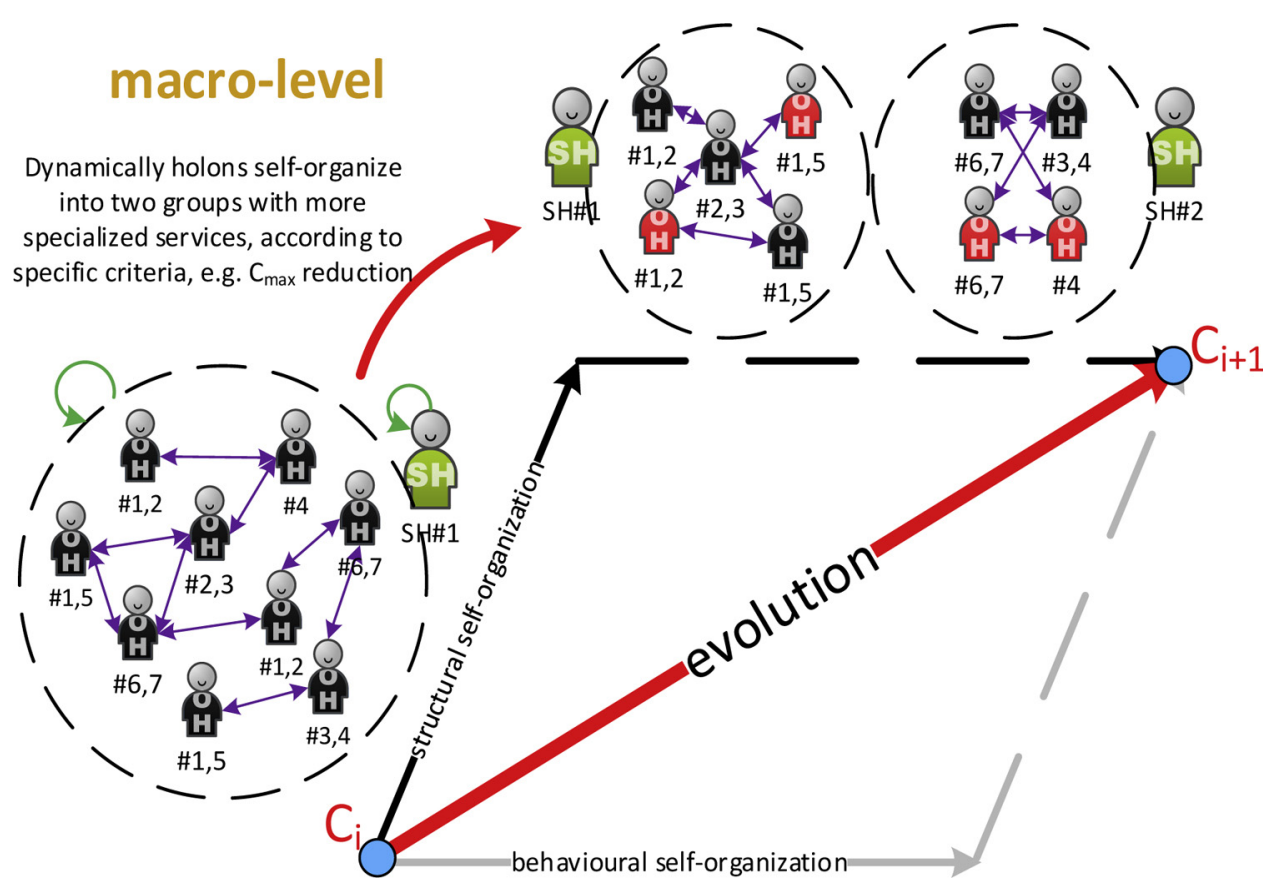

Fig. 6. Huge batch order detected by SHs.

system at a given point in time, the system detects this perturbation, through either the $\mathrm{SH}$ holons due to a high demand in variation requests or the individual $\mathrm{OHs}$ due to a high number of variation proposals, as shown in Fig. 6. Realizing these new constraints, the system selects the most adequate structure. In the present example, the $\mathrm{SH}$ detects the perturbation using its known algorithms, e.g. a Particle Swarm Optimization or Genetic Algorithm for resource allocation.

This example culminates with the creation of two groups, each supervised by a $\mathrm{SH}$ and managing a batch of orders, i.e. the current and the new one.

A pertinent question is how do the $\mathrm{ADACOR}^{2}$ holons structurally self-reorganize? Drawing inspiration from the social behaviour of schools of fishes and flocks of birds, it is concluded that they work very well as a group, maintaining the system equilibrium and, e.g., avoiding predators [24], which in this case are external system perturbations. The inexistence of any central authority regulating this global behaviour makes this mechanism even more robust and powerful.

$\mathrm{ADACOR}^{2}$ holons follow the same basic principles as swarms, constantly trying to build cohesive groups, maintaining distances and imposing crowd management. However, a central authority, which is lacking in nature, was intentionally introduced in $\mathrm{ADACOR}^{2}$ as it can increase optimization levels. In fact, this was already suggested in the work by Koestler, when he stated that the creation of stable intermediary states in the holonic holarchy introduces high levels of optimization. These higher level holons supervise a set of $\mathrm{OHs}$ which they try to optimize, e.g. optimizing schedules by coordination and synchronization of the groups' holons, and where it is possible to endow each group with a set of services as diverse as possible, creating the possibility to attend to a wider set of requests or to aggregate holons with similar services, creating specialized groups.

\subsection{Downward and upward causation}

The role of the two components in the self-organization mechanism needs the correlation of the effects between these two levels to be considered, i.e. the effect of the interrelation between behavioural and structural self-organization: the change in the behaviour of one holon will drive the system to evolve, then a new emergent global behaviour emerges, as it can be seen in Fig. 7. In a similar way, an evolution in holon behaviour occurs after a structural self-organization. A graphical explanation is presented in Fig. 7 using Coleman's boat analogy [36], which explains these interrelations in detail.

In brief, a change in the structural relations between holons implies a downward causation which will change the behaviour of individual holons, while a change in the behaviour of holons at micro-level may imply an upward causation that affects the structure of the relations between holons.

\section{Details of the self-organization model}

The self-organization model is translated to the architecture of individual $\mathrm{ADACOR}^{2}$ holons by considering a self-organization module inside the decision component of the holon, as illustrated in Fig. 8. This module addresses two types of self-organization components and comprises primarily the monitoring, discovery, reasoning, learning, nervousness stabilizer and dispatcher components.

The discovery and monitoring modules are supported by a database and their output feeds the reasoning and learning engines that process the data using a rule-based engine. This process is responsible for discovering new opportunities to evolve, as well as deciding the best way to evolve. The monitoring module is responsible for gathering information about the state of the system, e.g. analysing the exchange of messages or sensing the

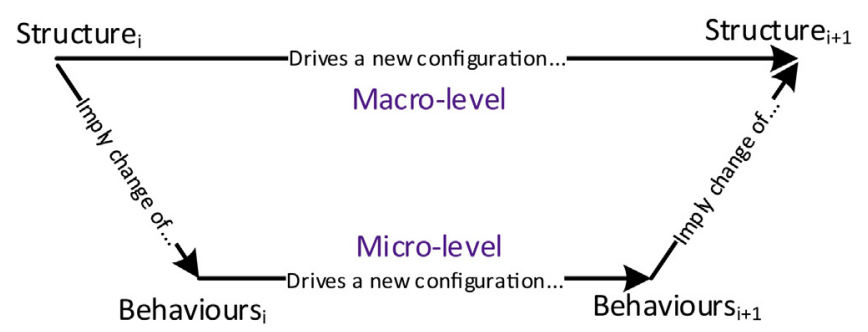

Fig. 7. Coleman's boat view in $\mathrm{ADACOR}^{2}$. 


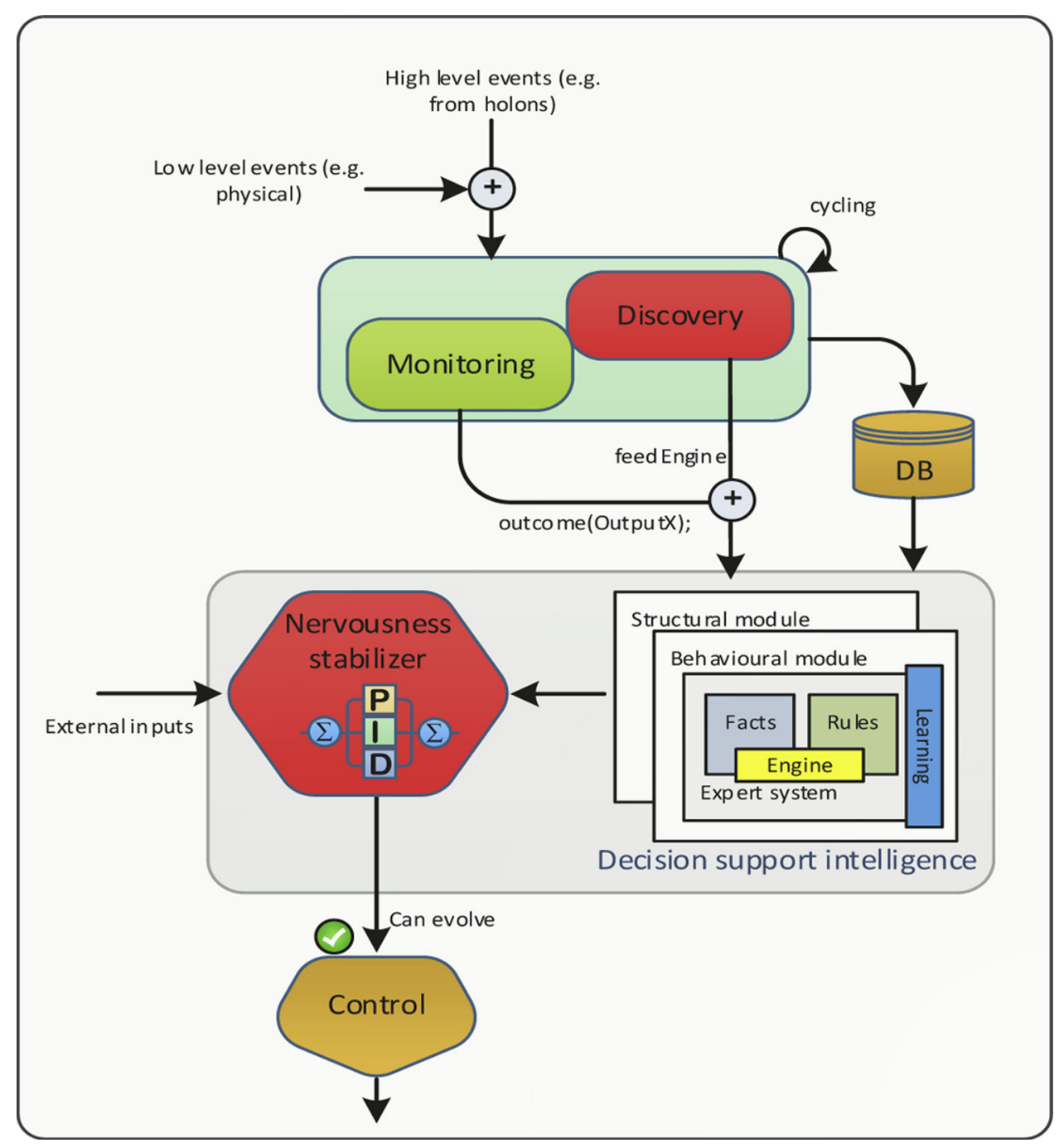

Fig. 8. Self-organization model in the $\mathrm{ADACOR}^{2}$ holon architecture.

holons' physical signals. In contrast, the discovery module is responsible for querying the holonic platform to find upcoming events, such as the presence of new holons, which can imply the appearance of a new holarchy. The output of both modules will feed the reasoning with facts that are later executed with a set of rules that composes the reasoner.

The reasoning module comprises two sub-modules, each containing the current rules for each type of self-organization, i.e. one for behavioural and the other for structural. In brief, when the monitoring and discovery modules issue new information, it is evaluated in the two reasoners' modules. Each of the reasoners provides outputs that are later evaluated by the respective nervousness stabilizer. The nervousness stabilizer prevents the holon from falling into chaotic behaviour.

\subsection{Reasoning and learning}

The learning capabilities, complementary to the reasoning module, play a crucial role in the self-organization model, supporting the generation, removal or adaptation of knowledge. In particular, learning is important in two distinct phases of selforganization:

(i) identification of opportunities to evolve and

(ii) defining how to evolve.
Different constraints impose different types of learning techniques and for this $\mathrm{ADACOR}^{2}$ uses social learning for the propagation of new behaviours among the holons and the propagation of accumulated knowledge from PHs to THs using a pheromone-like mechanism, as defined in the ADACOR architecture [19]. This mechanism preserves the same principals encountered in nature, as the closer a holon is to the epicentre of propagation, the more affected it will be, the impact being attenuated for the outer holons. This propagation has a gradient decay from its epicentre and also propagates to outside its holarchy, where some actions can be undertaken to check the status of the event.

The propagation of events to outside the starting holarchy raises a complementary issue that is related to the reliability of the warning propagation. To cope with this and check to what extent a holon can trust the information received, individual $\mathrm{ADACOR}^{2}$ holons use a trust-based mechanism to evaluate the inputs from other holons and assert if the information/behaviour is of value or not.

A second example of a learning technique based on rewards, i.e. reinforcement-based mechanisms, is used as a means to evaluate past evolution decisions. In this way, bad past decisions will have a negative impact on the future choice for the same decision contrary to a good result that will have a positive impact.

It is important to note that the learning mechanisms to be embedded in individual holons should be kept as simple as possible, as it is in biology. Nevertheless, more complex learning 
mechanisms can be developed, which can potentiate the learning module even more.

\subsection{Nervousness stabilizers: a way of controlling chaos}

One of the main problems that could appear in self-organized distributed autonomous systems is chaotic system behaviour where holons are continually changing their behaviour or enter a continuous cycle of constant evolution/adaptation. To cope with this issue, each individual $\mathrm{ADACOR}^{2}$ holon has a built-in stabilization mechanism, comparable to car shock absorbers, to avoid instability firstly in the holons and secondly in the system. By introducing these stabilizers, the system operates in such manner that it is pushed to its limits by enhancing the self-organization and chaotic principles, but always remains under control. A twolayer approach is used, as illustrated in Fig. 9, where the first one regulates each self-organization module individually and the second one merges the two, deciding which emergent behaviour will produce the most valuable evolution.

Some of the typical approaches to calm the holons' desire to change can be controlled by restricting the number of changes within a specific time frame, only allowing the entities to change at pre-defined intervals or by setting the exploration/exploitation threshold [37]. In this work, an innovative technique, inspired from the classical control theory, namely the Proportional, Integrative and Derivative (PID) controller, was used as the stabilization mechanism. The following three parameters are those of the controller: the proportional part $\left(K_{p}\right)$ that adjusts the reaction time to the perturbation trigger, the integrative part $\left(K_{i}\right)$ that regulates the accepted error of the desired goal, and the derivative part $\left(K_{d}\right)$ that sets the responsiveness to reach the goal (as in Eq. (1)):

$f(t)=K_{p} e(t)+K_{i} \int_{0}^{t}(\tau) d \tau+K_{d} \frac{d}{d t} e(t)$

Similarly, in ADACOR ${ }^{2}$, these three parameters regulate the selfcontrol of holons, where $K_{p}$ defines the time from which the holons start to react to triggers, allowing them to overcome momentary perturbations or to gather more information about the perturbation, e.g. in $\mathrm{OH}$, to start the self-organization mechanism only after the whole set of new allocation requests has been collected. In the PID controller analogy, a high value of $K_{p}$ will drive the system to respond faster to perturbations, which could lead the system to behave in an unstable manner, while a low value will not enable the system to reach the desired goal. The same cautions must also be taken into account when selecting the time after which the holons will react to plan deviations. A high value may drive the holons to be constantly in an adaptation mode while a low value might drive them to never react. Secondly, $K_{i}$ describes the minimum acceptable improvement of the solution found (by each self-organization mechanism) that can be considered as enough to permit the use of the changes. In the PID analogy, the $K_{i}$, or the integrative part, is responsible for the elimination of the error in steady state, i.e. going as close as possible to the desired goal. Since the goal of the ADACOR ${ }^{2}$ holons is to improve themselves always as possible, they're considered as moving a goal, which is a maximization function. In such way, the $K_{i}$ parameter acts as the minimum acceptable improvement of the actual goal. Lastly, $K_{d}$ defines how fast the solution must be found, acting as a limit to find solutions, after which the most acceptable ones are considered. In the PID analogy, the $K_{d}$ parameter helps to improve the settling time and increases system stability. Mimicking this to the nervousness controller, one will find that this parameter acts like the time limiter for a given holon to adapt. After this time, the holons will stop adapting and thus calm down its behaviour. The cautions to set this parameter regards the fact that a high value will enable a long adaptation time while a low value might not enable the holon to reach an acceptable goal value.

Another concern to be tackled in $\mathrm{ADACOR}^{2}$ is related to the fact that the self-organization process can be very time-consuming, namely in terms of the structural vector, which can be of major importance in large-scale systems in which the amount of information available increases exponentially. This important constraint is solved by taking a system snapshot, i.e. the current system state, whenever there is a disruptive event. This contextaware feature is complemented, at the end of the self-organization

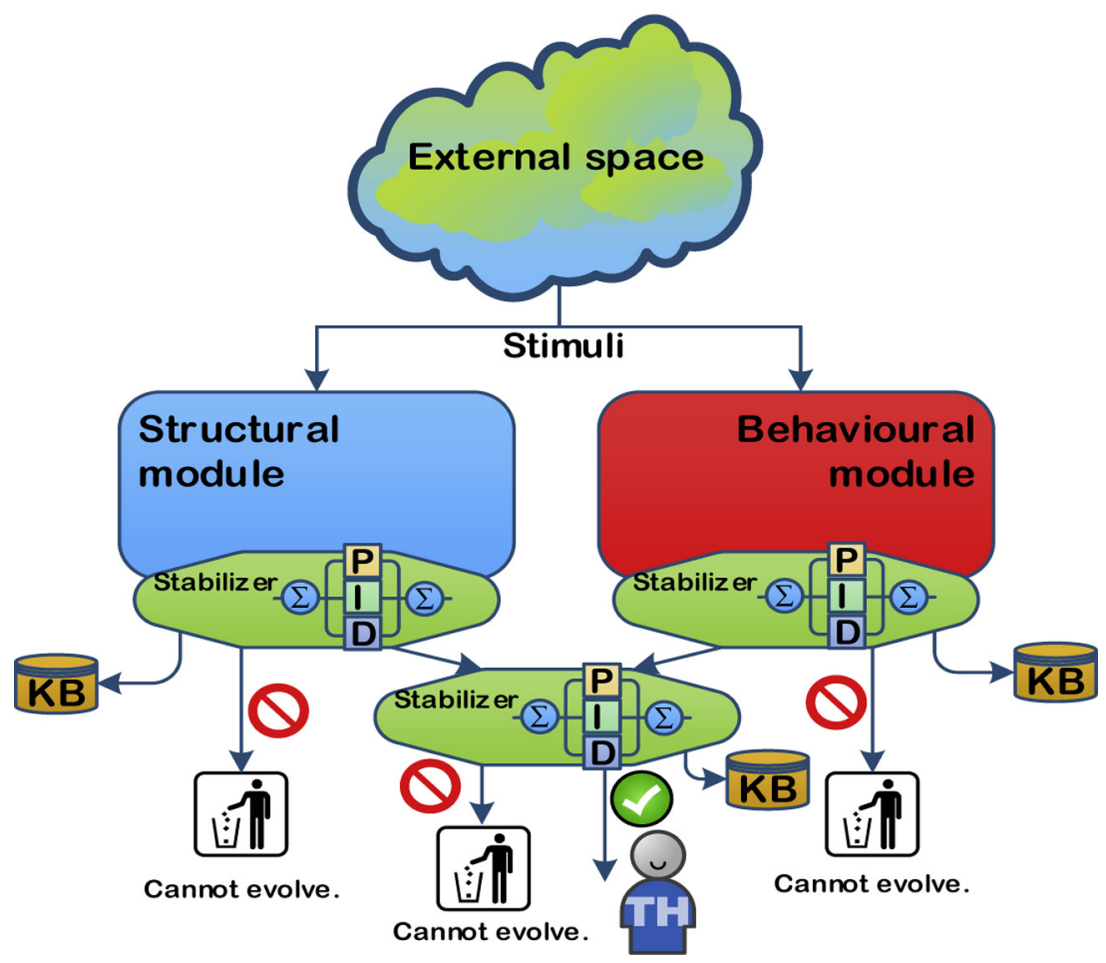

Fig. 9. Stabilization mechanism embedded in $\mathrm{ADACOR}^{2}$ holons. 
Table 1

Process plans for the catalogue of products.

\begin{tabular}{|c|c|c|c|c|c|c|c|}
\hline Sequence & B & $\mathrm{E}$ & $\mathrm{L}$ & $\mathrm{T}$ & A & I & $\mathrm{P}$ \\
\hline$\# 1$ & Loading & Loading & Loading & Loading & Loading & Loading & Loading \\
\hline \#2 & Axis & Axis & Axis & Axis & Axis & Axis & Axis \\
\hline \#3 & Axis & Axis & Axis & Axis & Axis & Axis & Axis \\
\hline$\# 4$ & Axis & Axis & Axis & r_comp & Axis & I_comp & r_comp \\
\hline \#5 & r_comp & r_comp & I_comp & L_comp & r_comp & Screw_comp & L_comp \\
\hline \#6 & r_comp & r_comp & I_comp & Inspection & L_comp & Inspection & Inspection \\
\hline \#7 & I_comp & L_comp & Screw_comp & Unloading & I_comp & Unloading & Unloading \\
\hline$\# 8$ & Screw_comp & Inspection & Screw_comp & & Screw_comp & & \\
\hline \#9 & Inspection & Unloading & Inspection & & Inspection & & \\
\hline \#10 & Unloading & & Unloading & & Unloading & & \\
\hline
\end{tabular}

process, with the actions taken and the results achieved (i.e. performance indicators), allowing the decision assessment afterwards. Additionally, this process can be enriched with data processing, e.g. data mining, to facilitate future events by allowing the system to find the best measures taken for similar events and start a warm self-organization process, i.e. it is not necessary to discover a new configuration, only to adapt a known one.

\section{Experimental results}

Two experimental simulation scenarios were developed to assess the proposed architecture. The behavioural self-organization was validated using the AIP-PRIMECA Flexible Manufacturing System (FMS) located at the Université de Valenciennes et du Hainaut-Cambrésis, and the structural self-organization, due to its particularities, was validated using a modified version of the AIPPRIMECA FMS, where the rigidity of the resources' position was removed and the conveyor system was replaced by Automated Guided Vehicles (AGVs).

\subsection{Description of the case study and system implementation}

The case study used in this work is presented in detail in [38]. In brief, the FMS is composed of six workstations linked by a conveyor system. The system is able to produce three products, namely the words "BELT", "AIP" and "LATE", each being composed of subassemblies (e.g. the product "BELT" is composed of the subassemblies "B", "E", "L" and "T"). The parts are assembled by the workstations according to a process plan designed for each part, as described in Table 1.

The workstations disposed along the FMS can perform a set of operations according to their skills, as illustrated in Table 2 (also including the processing time, in seconds, of each operation). As an example, the "Loading" operation can be executed by workstation $\mathrm{M}_{1}$ while the "Axis" operation can be executed by workstations $\mathrm{M}_{2}$ and $\mathrm{M}_{3}$, both with a processing time of $20 \mathrm{~s}$.

The proposed self-organized holonic system used multi-agent system technology, and more particularly, the Java Agent DEvelopment Framework (JADE) [39] to develop the agent-based

Table 2

Machine skills and processing times (in seconds).

\begin{tabular}{llllll}
\hline Operation & $\mathrm{M}_{1}$ & $\mathrm{M}_{2}$ & $\mathrm{M}_{3}$ & $\mathrm{M}_{4}$ & $\mathrm{M}_{5}$ \\
\hline Loading & 10 & & & & \\
Unloading & 10 & & & & \\
Axis & & 20 & 20 & & \\
r_comp & 20 & 20 & 20 & \\
I_comp & & & 20 & \\
L_comp & 20 & 20 & 20 & \\
Screw_comp & & & & & 5 \\
Inspection & & & & & \\
\hline
\end{tabular}

infra-structure, namely the behaviour of each individual ADACOR ${ }^{2}$ holon and the cooperation patterns designed. The holon's intelligence was embedded using a rule engine implemented with the Java Expert System Shell (JESS) platform [40].

In the real AIP-PRIMECA scenario, a particular holon type, named Conveyor System Holon (CSH), instantiated from an $\mathrm{OH}$, was developed to manage the routing and gate switching inside the conveyor system, providing some specific functions such as serving as an intermediary to manage the dispatching of the transportation orders to the available shuttles. The CSH uses the Jung tool [41] to support the dynamic parameter calculation in the nodes and also to provide display functionalities. The CSH has a Graphical User Interface (GUI), illustrated in Fig. 10, which provides real-time information regarding the location of the shuttles and the state of the workstations, according to a colourbased diagram.

The experimental tests were executed by considering the selforganized holonic agent-based solution developed using the JADE framework, to implement the logical control layer, which is populated by several instances of $\mathrm{TH}, \mathrm{OH}, \mathrm{PH}$ and $\mathrm{SH}$ holons. The implementation of holons having physical representation, e.g. OHs, requires the development of wrappers interfaces supporting the integration of the workstations presented in the AIP-PRIMECA case study. In this work, the virtual resource concept [42] was used to provide transparency in the intra-holon interconnection, encompassing the development of the implementation of the services at the server side (i.e. the resource) that will be invoked on the client side (i.e. the agent). In this way, the client ignores the details of the server implementation, and particularly for the control system perspective it is indistinguishable to be connected to an emulation platform or to a real system.

Having this in mind, a set of emulators are used to imitate each workstation presented in the AIP-PRIMECA case study, each one implemented as a JADE agent codified in Java language (the behaviour of each agent comprises a disturbance model defined for each workstation and presented in the next section).

\subsection{Analysis of the results of the behavioural self-organization experiment}

A battery of experimental tests, using a simulated AIP system, was conducted to validate the behavioural self-organization component, comparing different control approaches:

- Hierarchical approach, where a SH always provides optimized scheduling for the OHs.

- Heterarchical approach, running with no SH entity, i.e. each TH interacts directly and horizontally with the OHs.

- ADACOR approach, presenting the adaptive control approach balancing between hierarchical and heterarchical approaches in response to the occurrence of failures. 


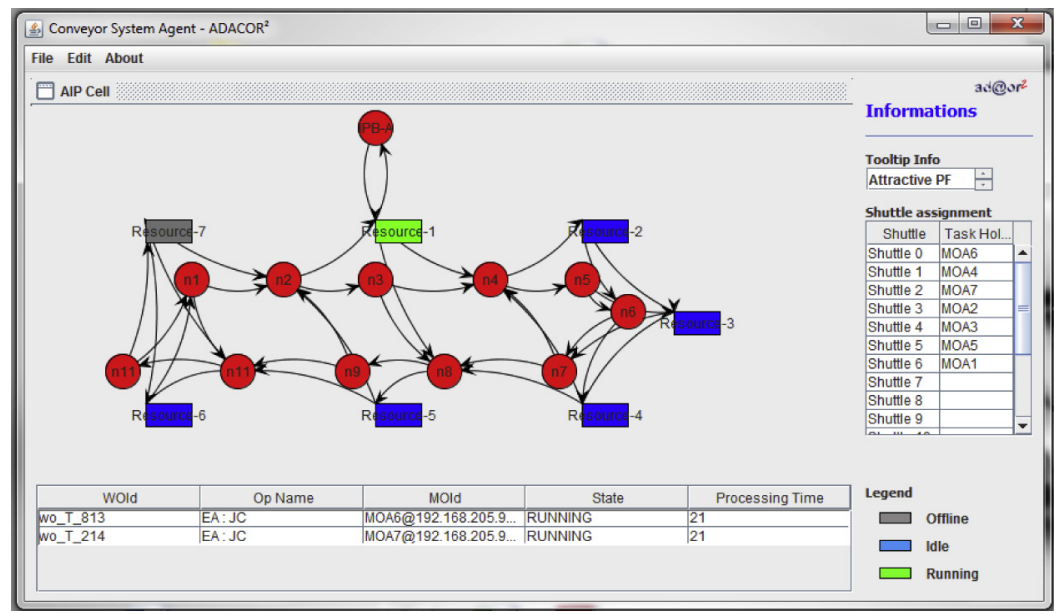

Fig. 10. GUI of the Conveyor System Holon.

Table 3

Results for the behavioural self-organization experiment.

\begin{tabular}{lllll}
\hline & Heterarchical & Hierarchical & ADACOR & ADACOR $^{2}$ \\
\hline $\begin{array}{c}C_{\max } \text { with no } \\
\text { perturbations (s) }\end{array}$ & 492.0 & 455.4 & 455.4 & 455.4 \\
$\begin{array}{c}C_{\max } \text { with } \\
\text { perturbations (s) }\end{array}$ & 551.5 & 563.5 & 535.4 & 522.8 \\
$\begin{array}{c}\text { Performance } \\
\text { degradation (\%) }\end{array}$ & 18.2 & 23.7 & 17.6 & 14.8 \\
\hline
\end{tabular}

- $A D A C O R^{2}$ approach, enabling the behavioural self-organization approach (in this work, by selecting new behaviours, such as those using a Potential Fields (PF) approach [43]).

Two experimental scenarios were considered: one with no perturbations and a second with perturbations. In the latter, failures were introduced in workstation $\mathrm{M}_{2}$ at a rate of $25 \%$ (corresponding to scenario \#PS12 of the benchmarking platform [38]), meaning that on average the resource will be unavailable every four work orders, guaranteeing determinism in the tests. In this situation, the work order affected and the subsequent ones need to be re-scheduled.

In this work, the comparison between the control approaches considers the production of a batch of "BELT" and "AIP" products, and aims at reducing the makespan $\left(C_{\max }\right)$. Table 3 summarizes the makespan achieved for each control approach taking the scenarios described into consideration.
As expected, the results show that in the scenario with no perturbations, the hierarchical-like approach achieved better results, i.e. more reduced makespan, than the heterarchical ones. In such situations, ADACOR and ADACOR ${ }^{2}$ exhibit the same results as the hierarchical approach due to the presence of supervisor holons which ensure optimized schedules for their subordinates, running in this manner in a hierarchical structure.

In the second scenario, with the possibility of perturbations occurring, all the control approaches analysed naturally suffered performance degradation, with the hierarchical approach suffering the most. On the contrary, ADACOR ${ }^{2}$ suffered the least degradation in performance, even less than the previous ADACOR and the heterarchical-like approach, since $\mathrm{ADACOR}^{2}$ enables the dynamic behavioural self-organization in each holon to cope with the occurrence of a perturbation, in this case selecting the PF behaviour for the re-allocation mechanism, evaluating which behaviour offers the most appealing outcome.

\subsection{Analysis of the results of the structural self-organization experiment}

To extract the full potential of the structural self-organization provided in $\mathrm{ADACOR}^{2}$, the trials assessed must allow shop-floor reorganization. This is not possible in the real AIP-PRIMECA FMS due to its physical rigidity. So, a modified system was considered, which assumed that workstations had moving capabilities in the sense that they can be unplugged from one working position and moved to another. A working position is defined as a place on the
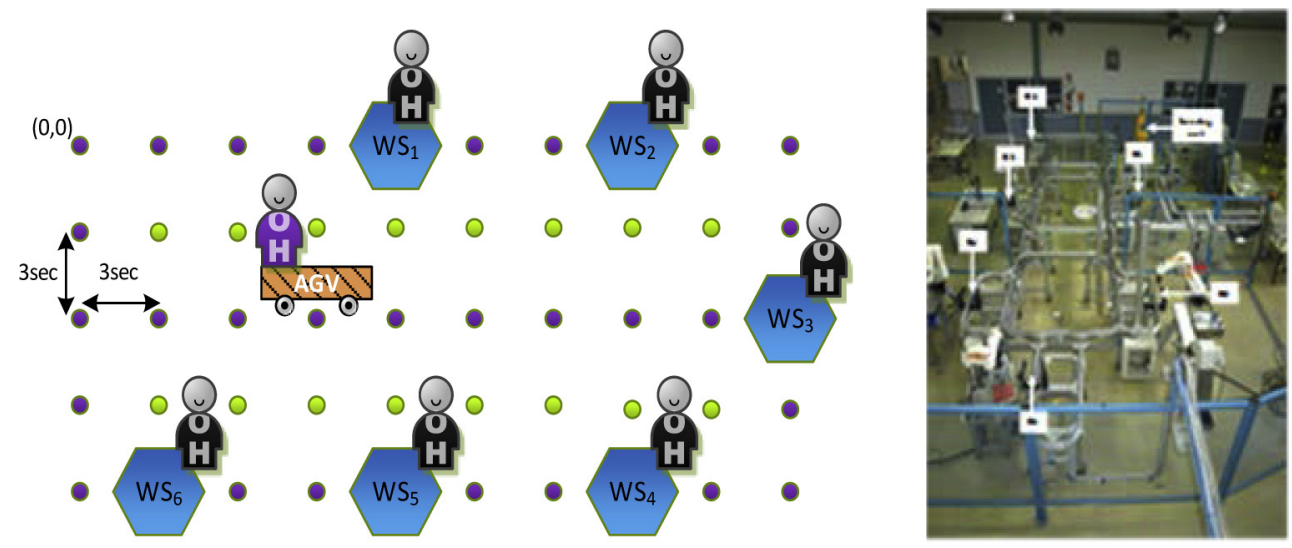

Fig. 11. Cell configuration test scenario (the real AIP cell is on the right). 


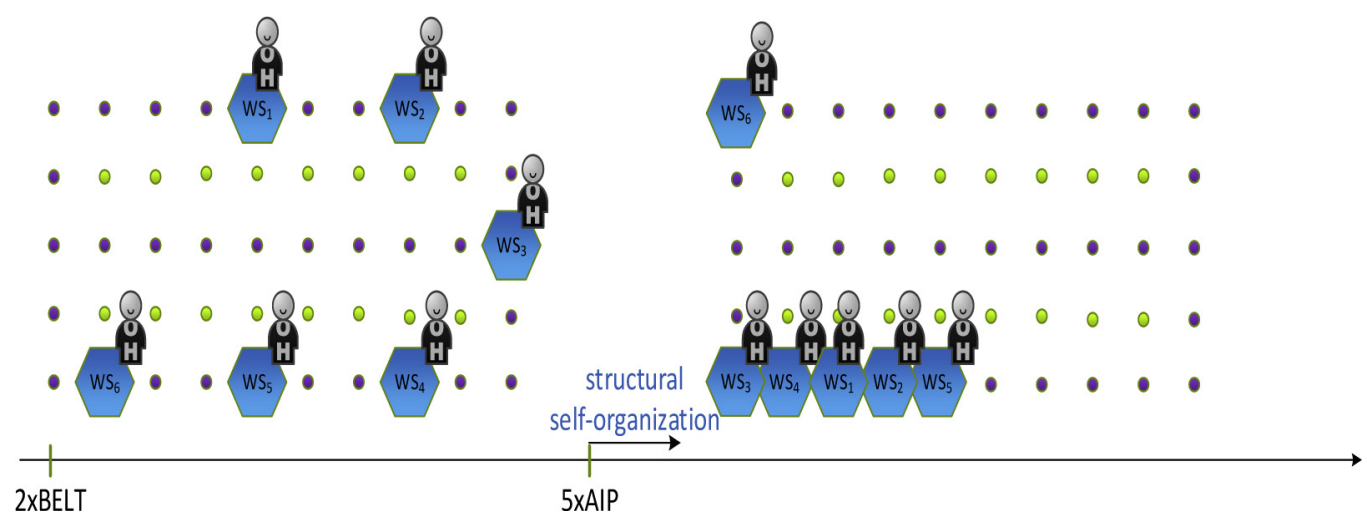

Fig. 12. Structural self-organization in practice.

shop-floor that is equipped with systems to support the normal functioning of the workstations, such as power, compressed air and communications. A second important change is the use of AGVs instead of the rail conveyor system. Fig. 11 depicts the conceptual view of the adapted system, where the purple points represent working positions and the green ones can only be used as routing paths. A fundamental issue to ensure coherence between this adapted system and the real one is the calculation of the transportation time that must be as close as possible between the two. For this purpose, it is assumed that moving from one point to another takes the AGV $3 \mathrm{~s}$ with a clear path. On the other hand, shifting resources not only depends on the distance to be travelled, but can vary from workstation to workstation due to size, weight and the complexity of cables.

With this in mind, as in the previous experiment, the ADACOR ${ }^{2}$ holons were deployed in the production control system, with workstations being mapped as $\mathrm{OHs}$ and each AGV as a specialized transporter instantiation of the $\mathrm{OH}$ (see Fig. 11). The CSH is no longer used here.

The experimental tests conducted in the present study assumed a level 3 structural self-organization since workstations can change place on the shop-floor. A big batch order was used by individual holons as the trigger to start the structural selforganization. Additionally, no central authority was used, making the structural self-organization complete in its magnitude, according to the concept of swarms found in nature. In brief, after receiving a batch of orders, every $\mathrm{OH}$ will start gathering information from other OHs, where resource queues, allocated work orders, processing times and actual location are exchanged. The procedure used in this example follows the principle where the most overloaded $\mathrm{OH}$ are the most critical. So, these resources should be fixed first, minimizing the transportation times between them. After allocating all the $\mathrm{OHs}$, each $\mathrm{OH}$ calculates the output results, i.e. the decrease/increase in $C_{\max }$, and shares it with the other OHs, also sending, in the case of a better solution, the KPIs and the new positions for each $\mathrm{OH}$. Finally, the best overall solution, found in all the OHs, is automatically assumed and used.

The system described initializes a manufacturing order to produce $2 \mathrm{x}$ "BELT" and after $120 \mathrm{~s}$ a new manufacturing order to

Table 4

Results for the structural self-organization experiment.

\begin{tabular}{llc}
\hline & Without & With structural \\
& structural & self-organization \\
& self-organization & \\
\hline$C_{\max }(\mathrm{s})$ & 1147.5 & 1112.0 \\
$\% \operatorname{transpTime~(\% )~}$ & 19.4 & 14.9 \\
$\operatorname{avg}($ order) (s) & 725.2 & 646.2 \\
\hline
\end{tabular}

produce $5 \mathrm{x}$ "AIP" appears in the system. This triggers a structural self-organization, as illustrated in Fig. 12.

Two scenarios were simulated and compared to evaluate the structural self-organization mechanism. The structural self-organization was disabled in the first scenario and enabled in the second. Several simulations were carried out for each scenario and the average values are presented in Table 4.

As can be observed by the analysis of the previous table, enabling the structural self-organization reduces the overall Cmax. This is mainly accomplished by the reduction in transportation times when the structural self-organization is enabled (reduction from $19.4 \%$ to $14.9 \%$ ). Additionally, a reduction in the time to process each individual order was observed.

The experimental results obtained show the merits of the structural self-organization approach to cope with severe, changing conditions. However, the experiments also showed new possibilities to improve the proposed algorithm, e.g. by considering optimization in the re-configuration of the resources during the structural self-organization process.

\section{Conclusions}

The complexity of systems has grown to unprecedented levels, triggering the need for new, more agile, robust and responsive control architectures. MAS and HMS play an important role in addressing this challenge by proposing a distribution of the processing capacity with several autonomous and cooperative entities.

Inspired from biology and evolutionary theories, this paper proposes an innovative control architecture called ADACOR $^{2}$, which considers the introduction of a two-vector self-organization mechanism: behavioural self-organization, found at micro-level, which allows the system to respond smoothly to perturbations, and structural self-organization, displayed at macro-level, which lets the system react more drastically. The introduction of selforganization properties in these autonomous entities and the overall system, may lead to some system nervousness, making the system more unstable and unpredictable. Since the objective is to push the system to its limits whilst maintaining its stability, nervousness stabilizers are also proposed to enhance the selforganization and chaotic principles, while maintaining the system under control.

This innovative architecture was tested, using simulations, on two different system topologies, namely the real AIP-PRIMECA system and a modified version of this system where transportation was achieved using AGVs and the workstations could move. In both scenarios, ADACOR $^{2}$ has been proven to display a better overall performance, in this case a lower $C_{\max }$, than the other control approaches tested. Consequently it is less affected by the 
occurrence of unexpected perturbations, frequent in industrial environments.

Future work will deal with merging the two self-organization mechanisms, by means of the second layer of the nervousness stabilizer, and considering the deployment of more powerful mechanisms for the behavioural and structural self-organization vectors. Additionally and despite this being a simulation validation approach, a more practical implementation is also envisioned by changing a parameter in the start of the agents, which control the real cell through ModBus communication commands.

\section{References}

[1] H. ElMaraghy, T. AlGeddawy, A. Azab, W. ElMaraghy, Change in manufacturing research and industrial challenges, in: H.A. ElMaraghy (Ed.), Enabling Manufacturing Competitiveness and Economic Sustainability, Springer Berlin Heidelberg, Berlin, Heidelberg, 2012, pp. 2-9.

[2] R.N. Nagel, R. Dove, S. Goldman, K. Preiss, Iacocca Institute of Lehigh University, United States, Department of Defense, Office of Managing Technology. 21st Century Manufacturing Enterprise Strategy, Iacocca Institute, Lehigh University, Bethlehem, PA, 1991.

[3] National Research Council (U.S.), Visionary Manufacturing Challenges for 2020, National Academy Press, Washington, DC, 1998

[4] Y. Koren, F. Jovane, U. Heisel, T. Moriwaki, G. Pritschow, G. Ulsoy, H. VanBrussel, Reconfigurable manufacturing systems, CIRP Annals 48 (2) (1999) 6-12.

[5] J. Ferber, Multi-Agent System: An Introduction to Distributed Artificial Intelligence, Addison-Wesley Professional, 1999.

[6] S.M. Deen, Agent based manufacturing: advances in holonic approach, in: Advanced Information Processing, Springer, New York, 2003.

[7] M. Onori, J. Barata, R. Frei, Evolvable Assembly Systems Basic Principles, in: Information Technology for Balanced Manufacturing Systems, 2006, 317-328.

[8] H.A. ElMaraghy, Flexible and reconfigurable manufacturing systems paradigms, International Journal of Flexible Manufacturing Systems 17 (2006) 261-276. http://dx.doi.org/10.1007/s10696-006-9028-7.

[9] M. Wooldridge, An Introduction to Multi-Agent Systems, John Wiley \& Sons, 2002.

[10] A. Koestler, The Ghost in the Machine, Arkana Books, 1969.

[11] L. Monostori, J. Váncza, S.R.T. Kumara, Agent-based systems for manufacturing, CIRP Annals - Manufacturing Technology 55 (2006) 697-720. , http://dx.doi.org/ 10.1016/j.cirp.2006.10.004

[12] F. Maturana, W. Shen, D.H. Norrie, MetaMorph: an adaptive agent-based architecture for intelligent manufacturing, International Journal of Production Research 37 (1999) 2159-2173. , http://dx.doi.org/10.1080/002075499190699.

[13] W. Shen, F. Maturana, D.H. Norrie, MetaMorph II: an agent-based architecture for distributed intelligent design and manufacturing, Journal of Intelligent Manufacturing 11 (2000) 237-251, http://dx.doi.org/10.1023/A:1008915208259.

[14] H.V.D. Parunak, A.D. Baker, S.J. Clark, The AARIA agent architecture: from manufacturing requirements to agent-based system design, Integrated Computer-Aided Engineering 8 (2001) 45-58.

[15] S. Bussmann, K. Schild, Self-organizing manufacturing control: an industrial application of agent technology, in: Proceedings of the Fourth International Conference on MultiAgent Systems (ICMAS-2000), ICMAS'00, IEEE Computer Society, Washington, DC, USA, 2000, pp. 87-94.

[16] S. Bussmann, J. Sieverding, Holonic Control of an Engine Assembly Plant - An Industrial Evaluation, Proceedings of the IEEE International Conference on Systems, Man and Cybernetics, (2001), pp. 169-174.

[17] H. Van Brussel, J. Wyns, P. Valckenaers, L. Bongaerts, P. Peeters, Reference architecture for holonic manufacturing systems: PROSA, Computers in Industry 37 (3) (1998) 255-274.

[18] M. Fletcher, D. McFarlane, A. Lucas, J. Brusey, D. Jarvis, The Cambridge packing cell - a holonic enterprise demonstrator, in: V. Mařík, M. Pěchouček, J. Müller (Eds.), Multi-Agent Systems and Applications III, Springer Berlin Heidelberg, Berlin, Heidelberg, 2003, pp. 533-543.

[19] P. Leitão, F. Restivo, ADACOR: a holonic architecture for agile and adaptive manufacturing control, Computers in Industry 57 (2) (2006) 121-130.

[20] R.F. Babiceanu, F.F. Chen, Development and applications of holonic manufacturing systems: a survey, Journal of Intelligent Manufacturing 17 (2006) 111-131. http://dx.doi.org/10.1007/s10845-005-5516-y.

[21] P. Leitao, V. Marik, P. Vrba, Past, present, and future of industrial agent applications, IEEE Transactions on Industrial Informatics (2012) 1, http://dx.doi.org 10.1109/TII.2012.2222034.

[22] P. Vrba, P. Tichý, V. Mařík, K.H. Hall, R.J. Staron, F.P. Maturana, P. Kadera, Rockwell automation's holonic and multiagent control systems compendium, IEEE Transactions on Systems, Man, and Cybernetics - Part C: Applications and Reviews 41 (2011) 14-30. , http://dx.doi.org/10.1109/TSMCC.2010.2055852.

[23] P. Leitão, Agent-based distributed manufacturing control: a state-of-the-art survey, Engineering Applications of Artificial Intelligence 22 (7) (2009) 979-991.

[24] P. Leitão, J. Barbosa, D. Trentesaux, Bio-inspired multi-agent systems for reconfigurable manufacturing systems, Engineering Applications of Artificial Intelligence 25 (2012) 934-944. , http://dx.doi.org/10.1016/j.engappai.2011.09.025.

[25] S. Camazine, J.L. Deneubourg N.R. Franks, J. Sneyd, G. Theraulaz, E. Bonabeau, SelfOrganization in Biological Systems, Princeton University Press, 2001.
[26] Deneubourg, S. Aron, S. Goss, J.M. Pasteels, The self-organizing exploratory pattern of the argentine ant, Journal of Insect Behavior 3 (1990) 159-168. http://dx.doi.org/10.1007/BF01417909.

[27] K. von Frisch, The Dance Language and Orientation of Bees, The Belknap Press of Harvard University Press, Cambridge, MA, 1967.

[28] R.C. Eberhart, J. Kennedy, A new optimizer using particle swarm theory, in: Proc. Sixth Int. Symp. Micromachine Hum. Sci., 1995, 39-43.

[29] Hadeli, P. Valckenaers, M. Kollingbaum, H. Van Brussel, Multi-agent coordination and control using stimergy, Computers in Industry 53 (2004) 75-96.

[30] T. Murata, Petri nets: properties, analysis and applications, Proceedings of IEEE 77 (1989) 541-580. , http://dx.doi.org/10.1109/5.24143.

[31] B. Bauer, J. Müller, J. Odell, Agent UML: a formalism for specifying multiagent software systems, in: P. Ciancarini, M. Wooldridge (Eds.), Agent-Oriented Software Engineering, Lecture Notes in Computer Science, Springer, Berlin Heidelberg, 2001, pp. 91-103.

[32] C. Darwin, On the Origin of Species by Means of Natural Selection, or, the Preservation of Favored Races in the Struggle for Life, Cosimo Classics, New York, 2007.

[33] N. Eldredge, S.J. Gould, Punctuated Equilibria: An Alternative to Phyletic Gradualism, in: Models in Paleobiology, 1972, 82-115.

[34] P. Kettunen, A tentative framework for lean software enterprise research and development, in: P. Abrahamsson, N. Oza (Eds.), Lean Enterprise Software and Systems, Springer Berlin Heidelberg, Berlin, Heidelberg, 2010, pp. 60-71.

[35] J. Barbosa, P. Leitao, A.I. Pereira, Combining adaptation and optimization in bioinspired multi-agent manufacturing systems, in: IEEE International Symposium on Industrial Electronics, 2011, 1773-1778. , http://dx.doi.org/10.1109/ ISIE.2011.5984425.

[36] J.S. Coleman, Foundations of Social Theory, Belknap Press of Harvard University Press, Cambridge, MA, 1994

[37] J. Barbosa, P. Leitão, E. Adam, D. Trentesaux, Nervousness in dynamic self-organized holonic multi-agent systems, in: Highlights on Practical Applications of Agents and Multi-Agent Systems, Advances in Intelligent and Soft Computing, 2012, 9-17.

[38] D. Trentesaux, C. Pach, A. Bekrar, Y. Sallez, T. Berger, T. Bonte, P. Leitão, J. Barbosa, Benchmarking flexible job-shop scheduling and control systems, Control Engineering Practice 21 (2013) 1204-1225. , http://dx.doi.org/10.1016/j.conengprac.2013.05.004.

[39] F.L. Bellifemine, G. Caire, D. Greenwood, Developing Multi-Agent Systems with JADE, John Wiley, Chichester, England/Hoboken, NJ, 2007.

[40] E. Friedman-Hall, Jess in Action, Manning/Pearson Education, Greenwich, CT/ London, 2003

[41] J. O'Madadhain, D. Fisher, P. Smyth, S. White, Y.-B. Boey, Analysis and Visualization of Network Data using JUNG, unpublished preprint.

[42] P. Leitão, F. Casais, F. Restivo, Holonic manufacturing control: a practical implementation, in: L. Camarinha-Matos (Ed.), Emerging Solutions for Future Manufacturing Systems. IFIP International Federation for Information Processing, Springer US, 2005, pp. 33-44

[43] C. Pach, A. Bekrar, N. Zbib, Y. Sallez, D. Trentesaux, An effective potential field approach to FMS holonic heterarchical control, Control Engineering Practice 20 (2012) 1293-1309. , http://dx.doi.org/10.1016/j.conengprac.2012.07.005.

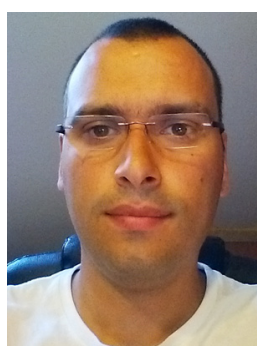

José Barbosa has a M.Sc. in Industrial Engineering at IPB, and is currently a Ph.D. candidate at the University of Valenciennes and Hainaut-Cambrésis (France). He is a researcher at Polytechnic Institute of Braganç, Portugal, participating in the EU FP7 ARUM project and in the past in the EU FP7 GRACE project. He is also invited teacher at the Department of Electrical Engineering of the Polytechnic Institute of Bragança. He has more than 20 papers published at international journals and proceedings of international conferences. His main research topics focus on the development of selforganizing and evolutive manufacturing control architectures following the holonic and multi-agent system paradigms enriched with biological inspired mechanisms. He is also member of the IEEE Technical Committee on Industrial Agents.

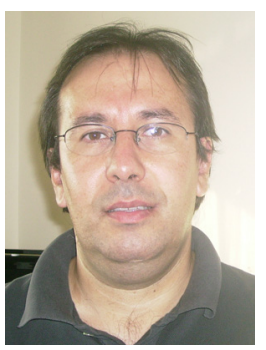

Paulo Leitão received the M.Sc. and Ph.D. degrees in Electrical and Computer Engineering, both from the University of Porto, Portugal, in 1997 and 2004, respectively. He is a professor at the Polytechnic Institute of Bragança, currently Head of the Department of Electrical Engineering, and member of the Artificial Intelligence and Computer Science Laboratory (LIACC) His research interests are in the field of industrial informatics, collaborative factory automation, reconfigurable production systems, intelligent supervisory control, agent-based and holonic control and bioinspiration engineering. He participate/has participated in several national and international research projects and Networks of Excellence, has published more than 130 papers in high-ranked international scientific journals and conference proceedings (peer-review), is coauthor of three patents and served as general co-chair of several international conferences, namely IFAC IMS'10 and HoloMAS'11. He is Senior member of the IEEE Industrial Electronics Society and currently the Chair of the IEEE Industrial Electronics Society Technical Committee on Industrial Agents. 


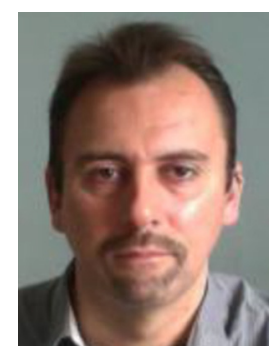

Emmmanuel Adam is an associate professor in Computer Science since 2003. His works concern holonic multiagent organizations, that is to say flexible, open and hierarchical organizations, where each element is free to choose its actions to reach its individual goal while being constrained by the necessity for the organization to reach its collective goal. The application fields are the transport simulation and the manufacturing systems management. He is a member of programme committees of international conferences (e.g. AAMAS, PAAMS) and reviewer for international journals (e.g. EAAI, IJPR, JIMS, COMIND).

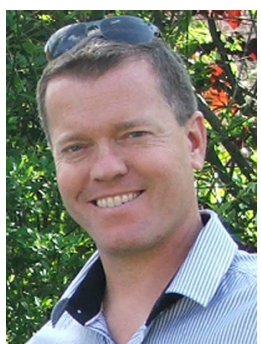

Damien Trentesaux is currently full professor at the University of Valenciennes and Hainaut-Cambrésis (France). His areas of interest concern the control and the optimization of discrete event systems (manufacturing, transport, logistics, and services) using multi-agent, bio-inspired and holonic models. He has supervised 12 Ph.D. thesis and is author and co-author of more than 100 peer reviewed publications in journals, books, and chapters of books and conference proceedings. He also co-chaired several special issues in journals edited by Elsevier, Springer and Lavoisier. He is a member of the IEEE Industrial Electronics Society (IES) Technical Committee on Industrial Agents. He is also member of the IFAC Technical Committee 5.1 on Manufacturing Plant Control. He belongs to the "board of editors" for the Elsevier Journal Engineering Application of Artificial Intelligence. 